\title{
Appropriate types of futures studies scenarios in health
}

\author{
Mostafa Hosseini Golkar ${ }^{1 *}$, Seyed Sepehr Ghazinoory², Fatemeh Saghafi ${ }^{3}$, \\ Mohammad-Rahim Eivazi ${ }^{4}$, Atousa Poursheikhali ${ }^{5}$, Reza Dehnavieh ${ }^{5}$
}

Received: 10 Jan $2016 \quad$ Published: 11 Dec 2017

\begin{abstract}
Background: Scenario is the primary method in futures studies, and thus its improper use can undermine the credibility and claims of the results. There are many scenario types, and here we aimed at understanding whether these scenarios are being used properly in the health field.

Methods: In this study, a combination method was used in 3 phases, and $8+2$ steps were considered to accommodate the needs of the health sector with capabilities of the main types of scenarios. One of the appropriate methods of futures studies was used at each step.

Results: Scenario planning has evolved along with futures studies paradigms. Trend-based scenarios, intuitive logic, and structural analysis approaches have had the most use in futures scenarios and health section. Quantitative techniques, which are close to the positivist paradigm, have been most widely used; however, participatory methods of futures studies paradigm have been used the least in the health sector.

Conclusions: Health scenario writing in its current state is targeting short- to medium- term futures and does not respond to all requirements. Thus, other backup methods should also be considered.
\end{abstract}

Keywords: Futures Studies, Health Section, Types of Scenarios, Methodology, Survey

Copyright@ Iran University of Medical Sciences

Cite this article as: Hosseini Golkar M, Ghazinoory SS, Saghafi F, Eivazi MR, Poursheikhali A, Dehnavieh R. Appropriate types of futures studies scenarios in health. Med J Islam Repub Iran. 2017 (11 Dec); 31:82. https://doi.org/10.14196/mjiri.31.82

\section{Introduction}

Studying the future is not only a need but also a necessity. Stepping into the future, as the late Pierre Wack (father of Shell's scenario planning) stated, "is somewhere in uncharted waters ahead" (1).

The future is profoundly different from the past (see Tofflers, 1996). This difference is particularly due to the accelerating rate of change and paradigm difference (Table 1) (2). This paradigm shift has divided the future, which was previously considered as same as the past, into 5 sections: near future (up to 1 year); the next short-term (up to 5 years); medium- term (up to 10 years); long- term (20 years); and far (up to the horizon of 100 years). Therefore, it is not possible to plan for the future only based on the experiences of the past, so to enter the new world, we need new tools, and futures studies (FS) are one of the efficient tools for this purpose. FS may become the domi-

Corresponding author: Mostafa Hosseini Golkar, mhq.golkar@gmail.com

1. Faculty of Management, University of Tehran, Tehran, Iran, \& Institute of Futures Studies in Health, Kerman University of Medical Sciences, Shahab, Kerman, Iran.

2. Department of Information Management, Faculty of Human Sciences, Modarres University, Tehran, Iran.

3. Faculty of Management, University of Tehran, Tehran, Iran

4. Department of Futures Studies, Imam-Khomeini International University, Qazvin, Iran.

5. Institute of Futures Studies in Health, Kerman University of Medical Sciences, Kerman, Iran. nant paradigm in futures thinking and a part of the culture and critical thought (3-6).

Wendell Bell discussed 9 major purposes or tasks of futures studies as follow: study of possible futures; study of probable futures; study of images of the future; study of the knowledge foundations of futures studies; study of the ethical foundations of futures studies; interpreting the past and orientating the present; integrating knowledge and values for designing social action; and increasing democratic participation in imaging and designing the future; and communicating or advocating a particular image of the future $(7,8)$.

Futures studies are a synthesis of science, art, and technology; they can be considered science because of their scientific accuracy, art because of using creativity to resolve the current crisis, and technology because of using

$\uparrow$ What is "already known" in this topic:

Scenario planning is a powerful tool for foresight in health sciences, and its main 3 approaches are trend-based, intuitive logic, and La-prospective scenarios.

\section{$\rightarrow$ What this article adds:}

There are different schools in the scenario field, and each has their own distinct approach. According to this paper, appropriate types of scenarios could be selected based on time horizon, scoping, and research needs. 


\begin{tabular}{cc} 
Table 1. Paradigm difference between the future and the past (2) \\
\hline Past & Futures \\
\hline Mechanical world & Organic world \\
Telescope view & Holographic view \\
Cartesian/Newtonian Paradigm & Knowledge-based paradigm \\
Simple & Cyber \\
Linear & Non- linear \\
Controllable & Non- controllable \\
Centralist & Network \\
Hierarchical & Cooperational \\
Limited communication & Extensive communication \\
Uniforms & Diverse \\
Predictable & Nonpredictable \\
Reductionist & Holistic \\
\hline
\end{tabular}

efficient tools to achieve goals (2).

Futures studies are interdisciplinary because they cross and combine various disciplines and have numerous rooted knowledge, particularly in the areas of engineering, basic sciences, social sciences, and philosophy of science. In other words, they are multidisciplinary because when entering a particular topic, they provide a holistic and macro view that goes beyond the scope of disciplines (8).

However, FS have become a historical evolution to present. At the beginning of the $20^{\text {th }}$ century, FS were just expert-driven and based on expert opinions about the future (prediction). However, prediction was not enough due to growth of the multi-specialized needs. Later, with the dominance of positivism, the trend studies attempted to be the main future study (forecast). Nonetheless, trend studies, due to the advent of trend-breaker events, did not support our growing needs. Therefore, planning based on the consensus of experts was considered in the 70s (foresight). Finally, in the late 20th century, the importance of target group's participation escalated to the current paradigm of futures studies according to the stakeholders' needs $(2,9)$ (Nowadays, foresight is more common in engineering sciences and FS are more common in social sciences.).

Similarly, with the development of FS paradigms, their methodologies also increased. Within the paradigm of prediction, intuitive methods, data mining, mind- mapping and interviews were prioritized. Within the paradigm of forecasting, quantitative methods, time series, and trend extrapolation were found to be the most important methods. Delphi, road map, and scenario were the most important techniques in the paradigm of foresight. In futures studies paradigm, all previous developed methods are implemented in workshops. Nevertheless, scenarios are unique in the methodology of futures studies. All methods of futures studies (more than 40 common methods $(2,10)$ ) can be used as input and introduction of scenarios, and the main output of FS processes are scenarios (8).

According to the Bain's annual survey (Bain \& Company's annual survey of management tools), statistics on the use of scenarios revealed that the scenarios tracked before 1990 were only found in $40 \%$ of firms, but their use increased to more than $70 \%$ in 2006 (11), and this trend continues to grow.

In brief, the proper use of scenarios indicates the level of maturity in futures studies. Moreover, the ability to provide comprehensive, provocative, and creative scenarios distinguishes professionals from amateur futurists.
There are some other general conditions on the use of futures studies methods that are often checked at the beginning of the projects in scoping, and then a suitable combined method will be derived (2). The most important considerations are as follow:

$\checkmark$ Research (or project) needs

$\checkmark$ Types of data input and output

$\checkmark$ The accuracy required in output

$\checkmark$ Time

$\checkmark$ Cost

$\checkmark$ Facilities

$\checkmark$ Readiness level (especially for implementation)

$\checkmark$ Absorption capacity

$\checkmark$ Organizational culture

$\checkmark$ Project implementation

$\checkmark$ Feedback, participation, etc.

Imprecision in selecting and combining methods will result in an incomplete project, so futures studies cannot attract protection or change their status.

From another perspective, however, area of futures studies is sensitive and important. Health has always been one of the most important factors (eg, in the Millennium Development Goals and in the Millennium Project, State of the Future Index (SOFI) method, health is the main topic of attention.).

Most futures studies processes are designed within the general framework with the frequent periods of feedback $(2,12)$, so here we selected a simple model of health care that fits the same pattern (Fig. 1) to derive the futures needs of health. These needs in the macro level are the need for description, exploration, norms, planning, etc. (Diagram 1).

The research questions are as follow: (1) What is the scenario maturity level (as the primary method and index of FS) in the health field?

(2) Are the scenarios used appropriately?

(3)Which types of scenarios are considered more in the field of health?

The improper use of scenarios can undermine the credibility and claims of the results of the futures studies.

In scenario planning literature, several approaches have been used to categorize their activities, most of which have been discussed by conventional research classified methods, such as explorative- normative, qualitative-

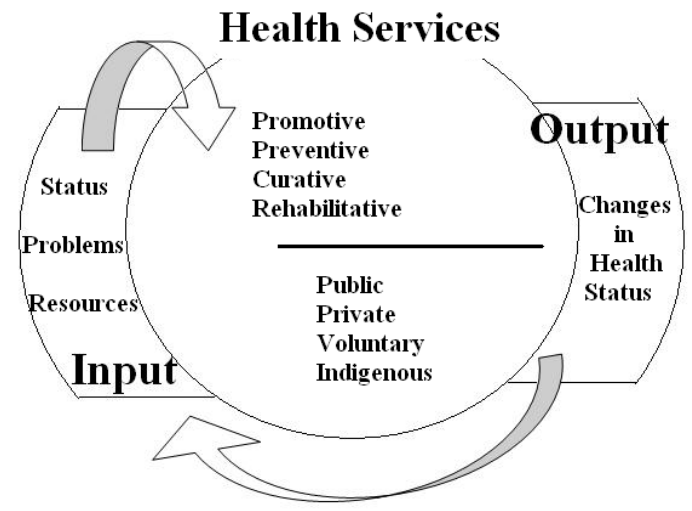

Fig. 1. Simple Health Care System Model (Adopted from Jonas's System Model (1952-2007)) (27) 


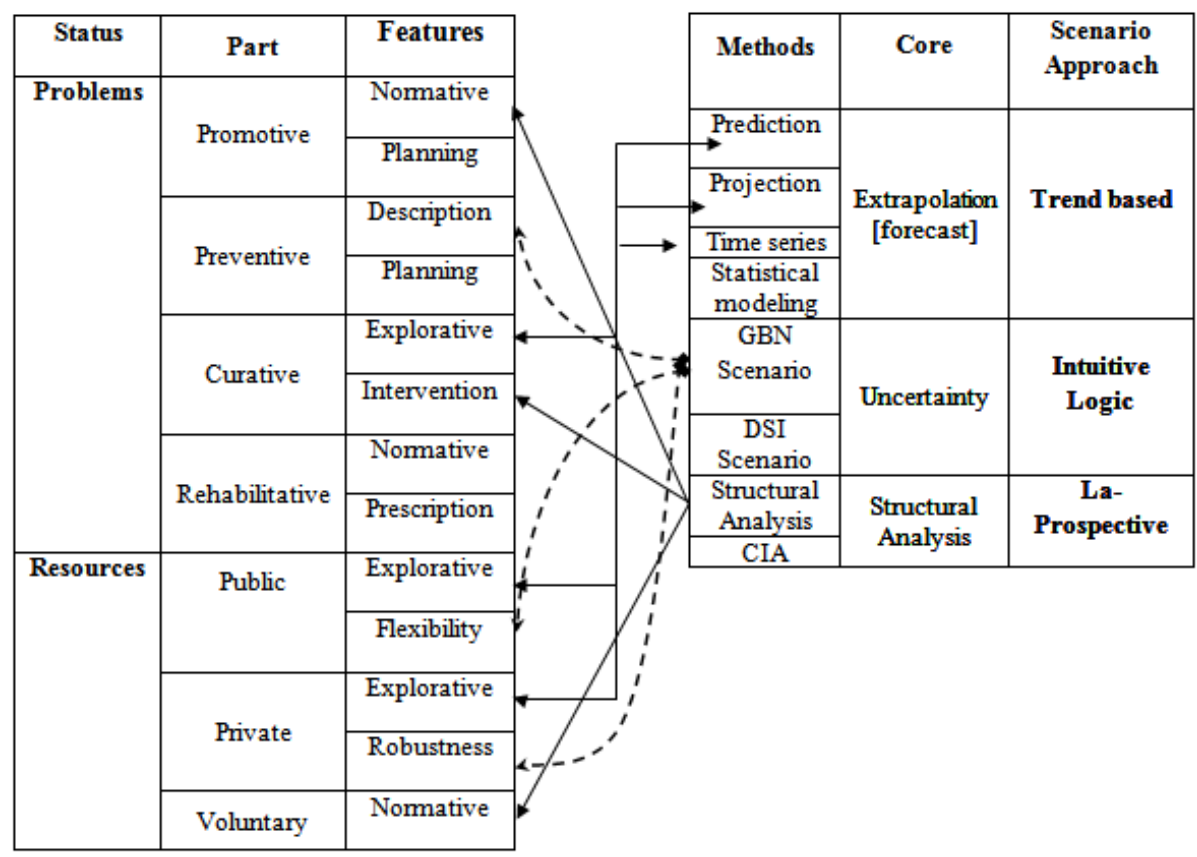

Diagram 1. Comparison of health needs \& types of scenarios (Note: Only important connections are shown.

For example, GBN approach has been used in almost all listed cases and in several investigations, however, because of focusing on critical uncertainties and using intuitive logic, and GBN targeting long-term futures, its maximum capacity is to describe prevention needs, flexibility in financing from public resources, and robustness in private sources. Finally, GBN is incomplete to meet other needs.)

quantitative, expert-driven, data-driven, and creative participation.

Other divisions based on historical period from 4 generations of scenario planning in the English-speaking world are as follow:

1. Herman Kahn in the 50s and 60s suggested intuitive and story-telling to encourage the understanding of the effects of current decisions.

2. Pierre Walk in the $70 \mathrm{~s}$ and $80 \mathrm{~s}$ expressed the importance of alternative thinking and changing mental models.

3. Peter Schwartz in the 90 s and early 21 st century focused on Key uncertainty.

4. New generation with a focus on network scenario planning was developed.

At the beginning of the 21st century, which Wendell Bell called it a period of transition and deepening in the foundations and epistemology of futures studies (8), numerous attempts were made to classify and deepen the understanding of the range of scenario planning (Table 2).

These articles were presented with the same goal and reviewed the domain of scenario to achieve organization and understanding of these areas. For this purpose, various ways of thinking about the scenario actions were presented.

\section{Methods}

Futures studies are high methodical knowledge, and designing and applying new combination methods are among the strengths of futurists (10). From the perspective of futures research as instrumental knowledge, each pro- ject requires its own unique method.

The combination of methods should consider the balance among the uses of expert-driven, participatory, datadriven, and creative approach (Popper's Diamond Allegory) (13) and provide both description and appropriate prescriptions of the future.

In this study, we extracted a new method based on research needs using a combination of methods appropriate to each step of the project (Fig. 2).

General phases of the research are as follow:

- Scenario survey phase (Thesis) in 3 steps

- Health needs assessment phase (Parallel) in 4 steps

- Comparative analysis phase (Antithesis)

\section{Outline of Steps}

Thesis phase:

Step 1: Establishing the position of scenarios in the futures studies through descriptive analysis

Step 2: Typology of scenarios through survey

Step 3: Characterization of the types of scenarios through discourse analysis

\section{Parallel phase:}

Step A: Selecting a public model that represents health field in a major view through a panel of experts (Expert Panels have been held in 2 meetings using focus discussion group method and combining the following 10 members: 4 in health sciences, 2 in methodology, 2 in social sciences, and 2 in IT.)

Step B: Deriving the general features of each part of the public model through experts query

Step C: Prioritizing the general features based on the 


\begin{tabular}{|c|c|c|c|c|}
\hline $\mathrm{R}$ & Year & Author(s) & Title & Subject / Result \\
\hline 1 & 2003 & Van Notten et al $(14,15)$ & $\begin{array}{c}\text { Scenario development: a typology } \\
\text { of approaches }\end{array}$ & $\begin{array}{c}\text { Typology "of the scenario" based on (1) purpose; (2) } \\
\text { project and (3) content }\end{array}$ \\
\hline 2 & 2005 & Bradfield et al (16) & $\begin{array}{l}\text { The origins and evolution of } \\
\text { scenario techniques }\end{array}$ & $\begin{array}{l}\text { The historical approach design / development, scripting } \\
\text { three schools }\end{array}$ \\
\hline 3 & 2006 & Börjeson et al (17) & $\begin{array}{l}\text { Scenario types and techniques: } \\
\text { towards a user's guide }\end{array}$ & $\begin{array}{l}\text { Scenario technique Classification based on Amara's } \\
\text { model }\end{array}$ \\
\hline 4 & 2007 & Biggs et al (18) & $\begin{array}{l}\text { Linking futures across scales: a } \\
\text { dialog on multi-scale scenarios }\end{array}$ & Layout and content of the Scenario \\
\hline 5 & 2007 & $\begin{array}{l}\text { Bishop, Hines \& Collins } \\
\text { (19) }\end{array}$ & $\begin{array}{l}\text { The current state of scenario } \\
\text { development: an overview of } \\
\text { techniques }\end{array}$ & Comparative Study of methods \\
\hline 6 & 2008 & Wilkinson et al (20) & $\begin{array}{c}\text { Evolving practices in environ- } \\
\text { mental scenarios: a new scenario } \\
\text { typology }\end{array}$ & Methodology \\
\hline 7 & 2011 & Turturean (21) & $\begin{array}{l}\text { Classifications of foresight meth- } \\
\text { ods }\end{array}$ & Methodology \\
\hline 8 & 2014 & $\begin{array}{l}\text { Ramirez \& Wilkinson } \\
(22)\end{array}$ & $\begin{array}{l}\text { Rethinking the } 2 \times 2 \text { scenario } \\
\text { method: Grid or frames? }\end{array}$ & Design, content and the question from Scale \\
\hline
\end{tabular}

Key technologies analysis method

Step D: Extracting related future needs with regard to general features for each part.

\section{Synthesis phase:}

First step: Comparative analysis of health needs with scenario types

Result step: Recommending and prescribing proper type of scenario for each part of the health sector

Pathology steps: Analyzing the current situation of health sector's scenario

\section{Results}

Most theorizing and macro views to future scenarios fall into 3 categories: (1) studies showing the schools of scenarios $(16,23)$; (2) studies looking at backup methods of scenarios (19); (3) and studies on the logic of scenarios $(24,25)$.

According to the needs of our present study, we considered 3 common approaches to scenarios based on the process, the inputs, and outputs: (1) trend based scenarios; (2) structural analysis; and (3) intuitive logic.

Trend-based scenarios are well-founded on the extrapolation process. In these scenarios, a trend derived from past events will be extended to the future. This approach assumes that the basic elements of the future are as in the past and unchanged. Trend analysis, quantitative methods, time series, and some types of modeling are the main inputs of trend-based scenarios $(2,10)$.

Structural analysis is a powerful tool developed in the La-prospective school by Michel Godet et al. (26). Scenarios, which are based on structural analysis, use stakeholders and actors' analysis in their input and rank the

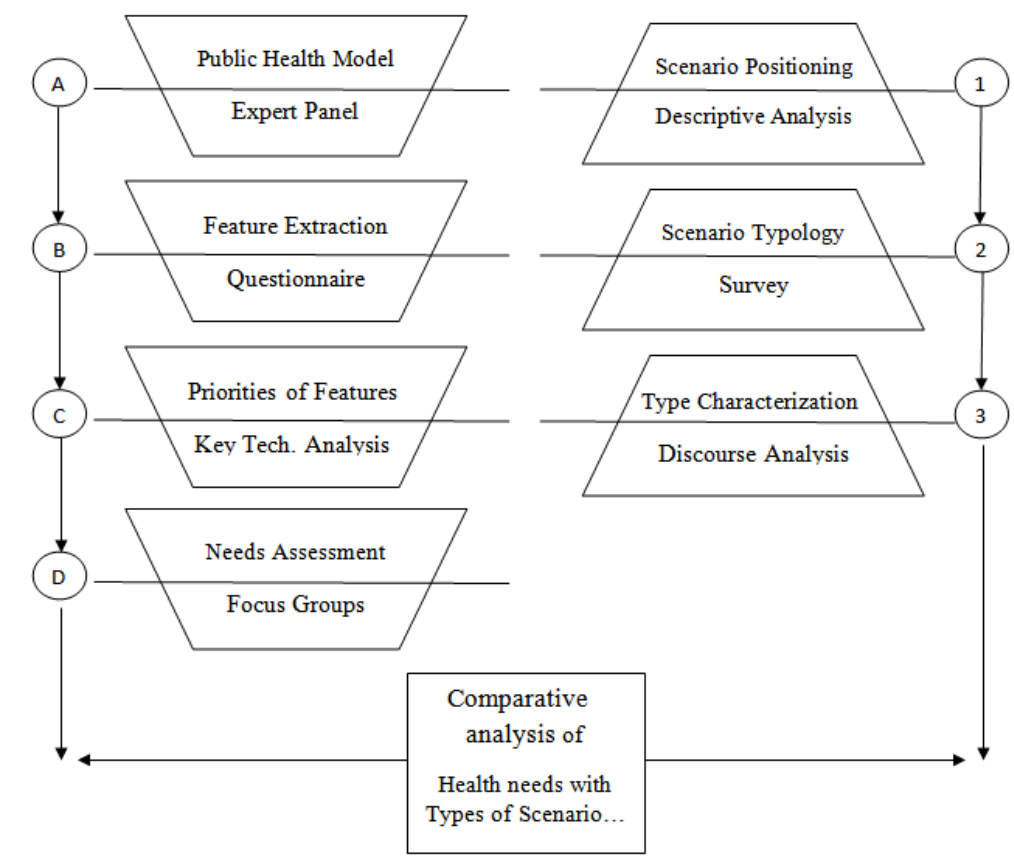

Fig. 2. Diagram of research methodology 
Table 3. Top $10 \mathrm{FS}^{\text {methods }}{ }^{1}$ in use (as surveyed in PubMed until 08, 2016)

\begin{tabular}{ccc}
\hline Top & Method & Frequency \\
\hline & & \\
1 & Prediction [forecast] & 170821 \\
2 & Simulation & 135125 \\
3 & Projection & 44991 \\
4 & Scenario & 34383 \\
5 & Time series & 20497 \\
6 & Structural analysis & 15009 \\
7 & Delphi & 6182 \\
8 & Data mining & 6030 \\
9 & Expert panel & 5727 \\
10 & Statistical modeling & 1502 \\
\hline${ }^{1}$ Methods as introduced in Millennium (10, also 2)
\end{tabular}

influencing factors and cross- impact analysis.

Intuitive logic is the main branch of scenario planning, which was started by Herman Kahn and was developed mainly by Royal/Dutch Shell School. Uncertainty is the core of scenarios, which is based on intuitive logic. These scenarios are aimed at identifying the uncertainty of their future through various processes and ultimately providing a narrative about the future with focusing on uncertainties $(23,24)$.

Content analysis and search in PubMed on futures studies methods related to the future's topics and issues revealed that trend analysis, intuitive logic, and structural analysis have, respectively, been used the most (Table 3).

\section{Discussion}

This study compared a simple model of health system and its needs with capabilities of futures studies methods. We focused on scenarios because they are the most important method of futures research (eg, see 8).

Evidence shows that the health sector has perceived the importance of scenarios, but neglected to fit the future needs of health (Table 4).

The main scenario approaches include trend-based, intuitive logic, and structural analysis. Trends based on past evidence pass on to future through judgment of experts. Various types of scenario production methods based on trends are used in the field of health. These methods are often quantitative and provide the ability to plan for the short-term to medium-term future.

Shell and Prospective, which can be called as English and French Schools, are pioneers of intuitive logic and structural analysis. Terminology and methodology of Shell School has grown in the field of business and the Prospective School in policy.

The intuitive logic has been used by many researchers in the field of health, but they simplified and reduced it. Thus, scenarios often lack the necessary components and elegance. In particular, less attention is paid to leading indicators, uncertainty extraction mechanism is unclear, and the logic of scenario development does not lead to decision-making.

Recently, the use of structural analysis scenarios is growing in the health field because of the free access to its software. However, the significance of backup workshops in these scenarios has not yet been understood.

Nowadays, health section scenarios are often descriptive
Table 4. Neglected FS methods in the health field (as surveyed in PubMed until 08 2016)

\begin{tabular}{ccc}
\hline $\mathrm{R}$ & Method & Frequency \\
\hline 1 & Road mapping & 1161 \\
2 & Visioning & 121 \\
3 & Multiple perspective & 37 \\
4 & Robust planning & 14 \\
5 & Mega trend & $<10$ \\
6 & CLA (Causal layered analysis) & $<10$ \\
7 & Trend impact analysis & $<10$ \\
8 & Morphological analysis scenario & $<10$ \\
9 & Back casting & $<10$ \\
10 & Workshop & $<10$ \\
\hline
\end{tabular}

and there are few normative scenarios (Although methods overlap, in some sources, they have been investigated in normative vs. explorative methods. (see 10, 13, 2). Quantitative techniques close to the positivist paradigm have been most widely used and participatory methods of futures studies paradigm have the lowest usage in the health sector. We have vacancies of illustrative scenarios that brought new in sights about the future (Table 4 and Diagram 1). Often the balance among data, creativity, expertise, and participation is not acknowledged. The current scenarios in health can only cover the short- and mediumterm periods and are incapable of intervening in the longterm.

\section{Conclusions}

All types of scenarios in futures studies are associated with their paradigm evolutionary, and each paradigm is responsive to certain needs. Thus, focusing on some methods of scenarios and ignoring the rest is our vulnerability.

It seems that futures studies in health still remain enclosed in the positivist paradigm. Thus, it is highly recommended to use trend-based scenarios in the short-term, structural analysis in the medium- term, and intuitive logic in part by the long-term needs.

Scenarios based on structural analysis are more appropriate in policy areas, as they consider the role of all key players and stakeholders and measure cross- impact and higher-order interactions.

To intervene in the long-term future in the health field, more attention should be paid to normative scenarios. Also, it is highly important to deepen future methods, such as causal layered analysis (CLA), which unfortunately has not yet been fully developed in the health section.

\section{Acknowledgments}

This article was derived from a $\mathrm{PhD}$ thesis $(8 / 3736.9)$ in Faculty of Management, Tehran University (2015-2016) and supported by HaMim Institute of Art \& Cultural Research, and also by Institute of Futures Studies in Health.

The authors would like to specially thank all participants for their kind contributions to this project; they are also grateful to Dr. Tahmours Hasangholi Pour, Dr. Saeed Khazaee, Dr. Mehdi Mohammadi, Dr. Somaye Noori Hekmat, Dr. Ghazaleh Taheri attar, Mohsen Keshavarz Turk, and Mohammad Mahdi Mowlaei for their insightful comments. 


\section{Conflict of interests}

The authors declare that they have no competing interests.
28. Tofflers. ' 5 billion futurists' in: Kurian GT, Molitor GTT. Encyclopedia of the Future. Simon \& Schuster and Prentice Hall International. 1996.

\section{References}

1. Wack P. Ж Scenarios: uncharted waters ahead. Harvard business review. 1985 Sep.

2. Hosseini Golkar M, Noori Hekmat S, Dehnavieh R, Poursheikhali A Encyclopedia of Futures Studies Methods. HaMim \& IFSH 2016. Also: Hosseini Golkar, M. Dastineh: Handbook of Futures Studies Methods. HaMim \& IFSH 2013.

3. Slaughter R. Recovering the future. Clayton: Graduate School of Environmental Sciences, Monash University, Australia, 1988.

4. Slaughter R. The foresight principle: Cultural recovery in the $21 \mathrm{st}$ century. Praeger Publishers; 1995.

5. Slaughter RA. Futures studies: From individual to social capacity. Futures. 1996 Oct 31;28(8):751-62.

6. Slaughter RA, Inayatullah S. Knowledge base of futures studies. Foresight International; 2005.

7. Bell W. The purposes of futures studies. The Futurist. 1997 Nov 1; 31(6):42.

8. Bell W. Foundations of Futures Studies: Human Science for a New Era: Values, Objectivity, and the Good Society. Transaction Publishers; 2011 Dec 31.

9. Kuosa T. Evolution of futures studies. Futures. 2011 Apr 30; 43(3):327-36

10. Gordon TJ, Glenn JC. Futures research methodology. Millennium Project of the American Council for the United Nations University. Version. 2003 Jul;2.

11. Rigby D, Bilodeau B. Bain's global 2007 management tools and trends survey. Strategy \& Leadership. 2007 Sep 11;35(5):9-16.

12. Voros J. A generic foresight process framework. Foresight. 2003 Jun $1 ; 5(3): 10-21$.

13. Popper R. Foresight Methodology. The handbook of technology foresight: concepts and practice. 2008 Mar 31:44.

14. Van Notten P. Scenario development: A typology of approaches. Teoksessa: CERI. Schooling for Tomorrow. Think Scenarios, Rethink Education.

15. Van Notten PW, Rotmans J, Van Asselt MB, Rothman DS. An updated scenario typology. Futures. 2003 Jun 30;35(5):423-43.

16. Bradfield R, Wright G, Burt G, Cairns G, Van Der Heijden K. The origins and evolution of scenario techniques in long range business planning. Futures. 2005 Oct 31;37(8):795-812.

17. Börjeson L, Höjer M, Dreborg KH, Ekvall T, Finnveden G. Scenario types and techniques: towards a user's guide. Futures. 2006 Sep 30; 38(7):723-39.

18. Biggs R, Raudsepp-Hearne C, Atkinson-Palombo C, Bohensky E, Boyd E, Cundill G, et al. Linking futures across scales: a dialog on multiscale scenarios. Ecology and Society. 2007 Jun 1;12(1):17.

19. Bishop P, Hines A, Collins T. The current state of scenario development: an overview of techniques. Foresight. 2007 Feb 27;9(1):525.

20. Wilkinson A, Eidinow E. Evolving practices in environmental scenarios: a new scenario typology. Environmental Research Letters. 2008 Dec 15;3(4):045017.

21. Turturean C. Classifications of foresight methods. Anuarul Institutului de Cercetari Economice" Gheorghe Zane"-Iasi. 2011 Jul $1 ; 20(2): 111$.

22. Ramirez R, Wilkinson A. Rethinking the $2 \times 2$ scenario method: grid or frames? Technological forecasting and social change. 2014 Jul 31; $86: 254-64$.

23. Chermack TJ. Scenario planning in organizations: how to create, use, and assess scenarios: Berrett-Koehler Publishers; 2011.

24. Van der Heijden K. Scenarios: the art of strategic conversation: John Wiley \& Sons; 2011.

25. Amer M, Daim TU, Jetter A. A review of scenario planning. Futures 2013;46:23-40

26. Arcade J, Godet M, Meunier F, Roubelat F. Structural analysis with the MICMAC method \& Actor's strategy with MACTOR method. Futures Research Methodology, American Council for the United Nations University: The Millennium Project. 1999-2010.

27. Jonas S, Goldsteen RL, Goldsteen K. An introduction to the US health care system. Springer Publishing Company; 2007 May 25. 